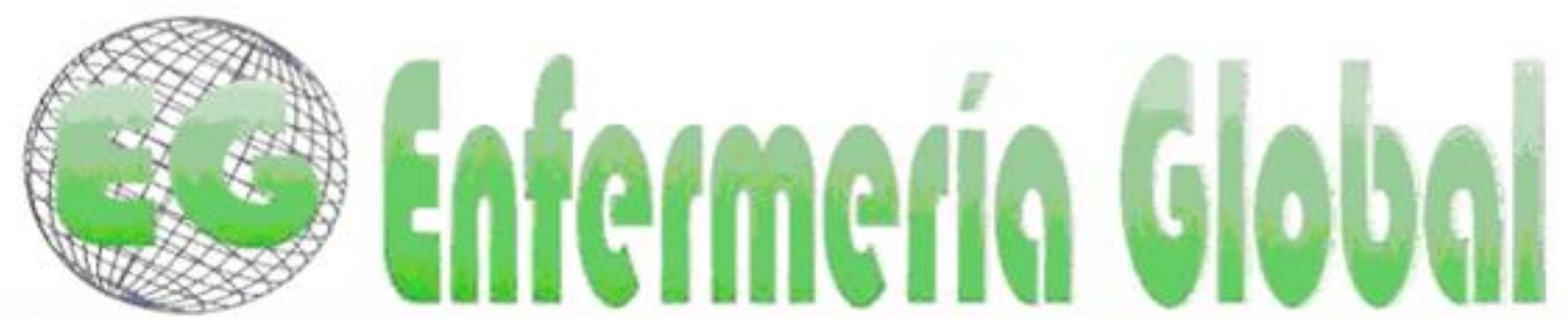

\title{
Estudio piloto. Memoria implícita, memoria explícita y deterioro cognitivo: evolución en el trastorno psicótico
}

Pilot study. Implicit memory and explicit memory and cognitive impairment: evolution in the psychotic disorder

*Pérez Moreno, Juan José *Romero García, Manuel *Salazar Gámez, Almudena ** Ortega Moreno, Beatriz

\author{
*Hospital Universitario Virgen del Rocio. Sevilla. E-mail: Juanjo_2k@hotmail.com ${ }^{* *}$ CRP San Juan de \\ Dios. Teruel. España. \\ Palabras clave: Memoria implícita; Memoria explícita; Deterioro Cognitivo; Trastorno Psicótico; \\ Esquizofrenia \\ Keywords: Implicit memory; Explicit memory; Cognitive impairment; Psychotic disorder; Schizophrenia
}

\section{RESUMEN}

En relación a los sistemas de memoria de nuestra investigación, clasificamos la memoria explícita, voluntaria, consciente; mientras que la memoria implícita no requiere la recuperación intencional o consciente.

Además del envejecimiento, las enfermedades psiquiátricas constituyen otras causas frecuentes de deterioro cognitivo; más específicamente, en el Trastorno psicótico están afectadas la memoria declarativa verbal (explícita), la memoria de trabajo y existen otros déficits cognitivos.

Metodología: El objetivo de este estudio consiste en analizar la evolución del Trastorno psicótico (en años) en relación a la memoria y al deterioro cognitivo, así como describir posibles asociaciones entre ambos tipos de memoria (implícita/explícita) y el deterioro cognitivo.

Se trata de un estudio descriptivo, observacional, transversal, formado por tres muestras de pacientes con Trastorno psicótico.

Resultados: Se obtienen proporciones medias mayores en los dos primeros grupos (0-5 años de evolución del Trastorno psicótico/ 10-15 años de evolución) que en el tercer grupo (25-35 años de evolución) para los resultados de memoria explícita. Respecto a la memoria implícita se observa la existencia de Priming en los tres grupos. Mientras que la puntuación positiva en el cribado de deterioro cognitivo es progresivamente mayor al aumentar los años de evolución de la enfermedad.

Conclusiones: Las personas con más años de evolución del trastorno psicótico presentan mayor deterioro cognitivo y puntuaciones medias menores en memoria explícita; mientras que la memoria implícita se mantiene estable independientemente de los años de evolución de la enfermedad. 


\begin{abstract}
In relation to systems of memory of our research report, we classify the memory explicit, voluntary, conscious; While the implicit memory does not require deliberate or conscious recovery.
\end{abstract}

In addition to aging, psychiatric diseases are other common causes of cognitive impairment; more specifically, the psychotic disorder affected the declarative (explicit) verbal memory, working memory and there are other cognitive deficits.

Methodology: The objective of this study is to analyze the evolution of the psychotic disorder (in years) in relation to memory and cognitive impairment, as well as describe possible associations between the two types of cognitive impairment and memory (implicitaexplicita).

It is a descriptive, observational, transversal study consisting of three samples of patients with psychotic disorder.

Results: Average proportions in the first two groups (0-5 years of evolution of the psychotic disorder 10 - 15 years of evolution) in the third group (25-35 years of evolution) to the results of explicit memory. Regarding the implicit memory is observed the existence of Priming in the three groups. While scoring positive in the screening of cognitive impairment it is progressively higher by increasing the years of evolution of the disease.

Conclusions: People with more years of evolution of the psychotic disorder have greater cognitive impairment and average scores lower in explicit memory; While the implicit memory stays stable regardless of the years of evolution of the disease.

\title{
INTRODUCCIÓN
}

La memoria permite mantener de forma actualizada diferentes elementos de información mientras los integramos entre sí ${ }^{(1)}$. El estudio de la memoria humana se ha convertido en un tópico de interés central estudiado desde múltiples enfoques ${ }^{(2)}$.

Fue Karl Lashley, en 1929, el que inició formalmente el estudio experimental de la memoria con su investigación llevada a cabo en ratas, encontrando que el deterioro en el desempeño en laberintos se correlacionaba más con la extensión de las lesiones cerebrales que con la localización de las mismas ${ }^{(3)}$. En 1949, Donald Hebb propuso que la memoria se encontraba conformada por subsistemas de memoria, además de sugerir el sustrato morfofisiológico de la memoria a largo plazo (4). Posteriormente, Larry Squire propuso en 1986 una taxonomía de la memoria, la cual la dividía en dos grandes subsistemas: la memoria declarativa y la no declarativa ${ }^{(5)}$. Casi al mismo tiempo, Endel Tulving propuso un modelo de la memoria que comprendía no dos, sino tres sistemas que trabajaban de forma conjunta: la memoria procedimental, la memoria semántica y la episódica. Para Tulving, la memoria a corto plazo y la episódica serían de tipo explícito, mientras que las otras serían implícitas ${ }^{(5)}$.

En relación a los sistemas de memoria que nos atañen en esta investigación, clasificamos en primer lugar la memoria explícita, voluntaria, consciente, que se evalúa normalmente mediante pruebas de recuerdo libre, recuerdo señalado y reconocimiento. Estas pruebas requieren la recuperación consciente de la experiencia almacenada. Este tipo de memoria se deteriora con la edad y se encuentra profundamente deteriorada desde las primeras fases de la enfermedad de Alzheimer $^{(6)}$. En claro contraste, la memoria implícita no requiere la recuperación intencional o consciente de la información codificada previamente y se evalúa de forma incidental utilizando una amplia variedad de pruebas indirectas o implícitas en las que no se hace ninguna referencia a la experiencia previa con los estímulos ${ }^{(6)}$. La forma habitual de mostrar la existencia de este tipo de memoria, consiste en señalar 
que existe priming perceptivo o de repetición, siendo el priming de repetición la mejor actuación con estímulos previamente presentados en comparación con estímulos nuevos ${ }^{(7)}$ que se expresa en pruebas implícitas en las que el procesamiento está determinado por las propiedades físicas de las claves del test, y un priming conceptual ${ }^{(8)}$, que se manifiesta en pruebas implícitas en las que se requiere un procesamiento semántico. La memoria implícita suele encontrarse preservada tanto en el envejecimiento normal con en las primeras fases del Alzheimer ${ }^{(6)}$.

A la hora de hablar de deterioro cognitivo en relación a la memoria implícita y explícita se debe contextualizar, pues persiste como un concepto mal delimitado y poco consensuado, que refleja una disminución del rendimiento de, al menos, una de las capacidades mentales o intelectivas siguientes: memoria, orientación, pensamiento abstracto, lenguaje, capacidad de juicio y razonamiento, capacidad para el cálculo y la habilidad constructiva, capacidad de aprendizaje y habilidad visoespacial (9). A menudo, solo el seguimiento clínico confirmará si estamos frente a una situación estable 0 , por el contrario, progresa a demencia. Una medida para evaluar el deterioro cognitivo hace referencia al test de Pfeiffer ${ }^{(10),(11)}$

Además del envejecimiento, otras causas frecuentes de deterioro cognitivo son las enfermedades psiquiátricas. Destacar la existencia de estudios recientes de trastorno de estrés postraumático (TEPT), los cuales sugieren que la codificación de un episodio dentro de un contexto de miedo genera representaciones diferentes respecto a la memoria implícita y explícita; indican que el implícito, pero no explícito, rastro de la memoria de un contexto temeroso de un episodio puede ser detectada a largo plazo ${ }^{(12)}$.

Más específicamente en aquellas patologías que abarcan el amplio espectro de la psicosis (en especial aquellas que corresponden a F1x.5, F1x.7, F20-29) (13); mencionar que la función mnésica está especialmente afectada en los pacientes con trastorno psicótico, y más si cabe en esquizofrenia, con un deterioro de la memoria explícita declarativa (verbal y espacial) así como de la memoria episódica. Estas alteraciones de la memoria se asocian con la cronicidad de la enfermedad y con la falta de respuesta al tratamiento ${ }^{(14)}$.

Existe una prueba (CVLT/TAVEC) que mide el rendimiento en la memoria verbal y detecta una posible disfunción neurocognitiva. Otro método para medir la memoria en esquizofrenia hace referencia a la utilización de un subtest del WAIS-III, Letras y números (LN), para medir memoria de trabajo verbal ${ }^{(15)}$. El DPT (Doors and People Test) es una medida neuropsicológica de memoria episódica (16). Existen una gran cantidad de medidas de memoria implícita; encontrándose que enfermos amnésicos, depresivos o esquizofrénicos que presentan una memoria explícita muy deteriorada, muestran una memoria implícita equivalente o casi equivalente a la de los individuos sanos $^{(17)}$. Las personas con esquizofrenia presentan un deterioro en el procesamiento de señales sociales como las expresiones faciales de emoción. La percepción de expresiones faciales es un proceso complejo que depende de una red neuronal distribuida dentro de las regiones implicadas en el procesamiento visual, cognitivo y afectivo; en un estudio se examinó el priming de repetición, se exploraron los procesos perceptivos visuales asociados a la percepción de la expresión facial en personas con esquizofrenia; como resultado el priming fue normal en los pacientes con esquizofrenia, pero, como era de esperar, la memoria de reconocimiento para las expresiones faciales estaba deteriorada ${ }^{(18)}$. En otro estudio se observó que los pacientes esquizofrénicos logran un priming tan alto como el de los controles en la 
tarea de decisión léxica; en contraste, sólo los controles mostraron priming significativo en la tarea de verificación de la categoría. Por lo tanto se concluyó que la esquizofrenia está asociada con un déficit específico en la memoria implícita conceptual ${ }^{(19)}$. Los déficits de memoria semántica en la esquizofrenia son profundos, pero no hay ninguna investigación comparando procesamiento semántico implícito y explícito en la misma muestra. Existe un estudio en el que se destaca el patrón único de actuación específica del priming semántico; finalmente, la eficacia del priming se discute en relación con el trastorno del pensamiento y según la duración de la enfermedad ${ }^{(20)}$.

Respecto a las pruebas de mayor uso para evaluar la memoria explícita se encuentran las clásicas pruebas de recuerdo y reconocimiento. En las primeras, se pide al participante que reproduzca el material presentado previamente en el mismo orden o en el orden que se desee (recuerdo libre). A veces, pueden proporcionarse señales que ayuden en la recuperación (recuerdo señalado). En las pruebas de reconocimiento se presentan todos los elementos que habían sido estudiados previamente durante la fase de estudio o codificación junto con otros estímulos nuevos para que distingan entre antiguos y nuevos. Las tareas experimentales de recuerdo y reconocimiento constan de dos fases: En la primera fase, los participantes estudian una serie de estímulos (por ejemplo, palabras, imágenes, sonidos, objetos). Tras un intervalo temporal variable (generalmente 3-5 minutos) en el que suelen realizar otra tareas (tarea distractora) consistente por ejemplo en decir nombres de personas, contar hacia delante o hacia atrás desde un determinado número, los participantes realizan la fase de prueba o test. En esta fase, se les pide que recuerden los estímulos que habían sido estudiados durante la fase de estudio (con o sin pistas) o que reconozcan en un conjunto, aquellos estímulos que habían sido presentados en la fase de estudio ${ }^{(15)}$.

En nuestro estudio hemos decidido emplear la prueba de compleción de raíces de palabras, ya que es una de las pruebas más utilizadas para evaluar el priming de repetición. También puede utilizarse esta misma tarea, modificando las instrucciones, para evaluar la memoria explícita. En este caso, la prueba se denomina recuerdo señalado de raíces de palabras. La utilización de la información estimular en la prueba implícita y en la prueba explícita tiene la ventaja de que lo único que cambia son las instrucciones proporcionadas por el experimentador a la hora de evaluar la memoria. La prueba consta de dos fases: una fase de estudio (codificación) y una fase de prueba. En la fase de estudio, se presenta a los participantes una serie de palabras para que realicen con ellas alguna tarea. Esta tarea puede consistir en procesar cada palabra de una manera superficial, perceptiva o léxica (haciendo referencia a la memoria implícita), o de una manera profunda procesando semánticamente el significado de la palabra (memoria explícita). Tras un breve intervalo temporal (5 min) durante los que los participantes realizan una tarea distractora, comienza la fase de prueba de memoria. En esta fase, se proporciona al participante series de raíces de palabras formadas por las tres primeras letras y se le pide que complete estas raíces con la primera palabra que le venga a la mente. Generalmente la mitad de las raíces de palabras presentadas proceden de la lista de estudio presentada al comienzo y las otras son nuevas ${ }^{(7)}$.

Varios estudios han sugerido que los procesos de reconocimiento de memoria podrían estar basados en proceso de memoria implícita incluso cuando los participantes son conscientes de la recuperación de la memoria, un fenómeno conocido como "reconocimiento implícito"; diversos procesos de memoria implícita 
están involucrados en diferentes formas de memoria de reconocimiento. Debe prestarse cuidadosa atención a la influencia de estos procesos implícitos en diversos tipos de pruebas de memoria ${ }^{(21)}$.

Es necesario destacar que la atención en la codificación de información juega un papel crítico y omnipresente en el rendimiento de la memoria explícita, pero su papel en el rendimiento de la memoria implícita (es decir, priming) es más variable: algunos (pero no todos) efectos del priming son reducidos por la división de la atención en la codificación. Una amplia variedad de trabajos teóricos y empíricos tiene como objetivo definir las características de los efectos del priming que no requieren atención en la codificación. Los resultados de un estudio sugieren que la diferenciación de papel de la atención dentro del priming inducido ocasiona costes y beneficios que son ligados a las diferencias en la competición de respuesta asociada a estos efectos ${ }^{(22)}$.

En cuanto a la memoria a corto plazo los hallazgos, a pesar de ser contradictorios, apuntan a un déficit tanto en el área de memoria visual como en la verbal en pacientes con esquizofrenia. En cuanto a la memoria a largo plazo, las conclusiones son aún menos concordantes ${ }^{(23)}$. De todos modos, el déficit en la memoria verbal declarativa episódica parece ser el hallazgo más consistente ${ }^{(24)}$.

En un estudio en el que comparan la memoria episódica verbal en pacientes con Trastorno bipolar y Esquizofrenia se encontró un declive cognitivo junto con la progresión del trastorno bipolar, mientras que el deterioro estaba ya presente en la etapa temprana de la esquizofrenia. Se hace hincapié en la importancia de estudiar nuevas estrategias terapéuticas, en particular, las técnicas de rehabilitación cognitiva como tratamiento prometedor para los pacientes psiquiátricos, incluso en las personas con discapacidad moderada ${ }^{(25)}$.

Uno de los déficits más comunes en los pacientes con esquizofrenia se da en la memoria de trabajo, cuyo alcance tiene un amplio efecto en la cognición. Sin embargo, enfoques anteriores del estudio de la memoria de trabajo en pacientes esquizofrénicos han utilizado tareas que requieren varios procesos de control cognitivo, por lo que es difícil determinar qué procesos cognitivos y neuronales específicos subyacen al deterioro de la memoria de trabajo. En un estudio realizado con controles sanos y pacientes con esquizofrenia se destaca que la inhibición deteriorada provocó un aumento de la dependencia del control de interferencia y redujo el rendimiento de la conducta ${ }^{(26)}$. Diversos estudios llegan a la conclusión de que al analizar el rendimiento cognitivo del grupo de pacientes esquizofrénicos según los años de evolución de la enfermedad pudieron observar que el deterioro cognitivo que existe en la esquizofrenia tiende a permanecer estable ${ }^{(27)}$.

En lo referente a la rehabilitación del déficit en memoria, nos hallamos en el campo en el cual ha habido menos intentos de abordaje específico ${ }^{(28)}$. Recientemente se han diseñado programas terapéuticos específico, como el REHACOP, programa de rehabilitación cognitiva en psicosis ${ }^{(29)}$. En los últimos años se han realizado ya trabajos en los que específicamente se estudia la aplicación y eficacia de la rehabilitación neurocognitiva en la esquizofrenia. Mientras que existen estudios (30) que demuestran un cambio en la activación cerebral asociado a la intervención neurocognitiva, precisamente sobre una función supuestamente deficitaria en la esquizofrenia, como la memoria operativa. 
Dada la emergente reconceptualización de la cognición en esquizofrenia (y otros trastornos psicóticos) como el reflejo de una anormalidad de base neurobiológica se sugiere que un deterioro en el control proactivo puede influir en el rendimiento en una amplia variedad de dominios cognoscitivos y por lo tanto puede representar un mecanismo común de contribuir a estos déficits ${ }^{(31)}$. Los pacientes con esquizofrenia muestran un pronunciado déficit en la memoria, en comparación con otros dominios. En particular, los pacientes con síntomas negativos, especialmente afectividad plano, tienen severos déficits de memoria y esto se asocia con peor calidad de vida y ajuste funcional. Esta condición es más frecuente en varones con esquizofrenia y puede relacionarse con la mayor prevalencia y severidad de los síntomas negativos. Dada su centralidad, la memoria debería ser un objetivo importante para la intervención en esquizofrenia ${ }^{(32)}$.

Para concluir es necesario precisar ciertos puntos clave en nuestra investigación:

- El deterioro cognitivo presente en el trastorno psicótico es severo y afecta especialmente la memoria declarativa verbal, la memoria de trabajo y ocasiona otros déficits cognitivos como la afectación de las funciones ejecutivas y la atención sostenida.

- $\quad$ Este déficit se asocia del siguiente modo ${ }^{(33)}$ :

> Los déficits de la memoria declarativa (explícita) y de la capacidad de atención sostenida afectan el funcionamiento social.

$>\quad$ Los déficits de las funciones ejecutivas, de la memoria declarativa (memoria explícita), de la memoria de trabajo y de la atención sostenida afectan el funcionamiento ocupacional ${ }^{(34)}$.

$>\quad$ Los déficits de las funciones ejecutivas, la memoria declarativa (memoria explícita) y la memoria de trabajo afectan a la capacidad de vivir independientemente.

Por lo tanto, en relación a nuestra investigación es necesario destacar que nuestro objetivo general consiste en analizar la evolución en el tiempo del Trastorno Psicótico en relación a la memoria y al deterioro cognitivo. Mientras que nos planteamos una serie de objetivos específicos como son: describir si existe correlación y de qué tipo en cuanto al deterioro cognitivo y la memoria explícita; describir si existe correlación y de qué tipo en cuanto al deterioro cognitivo y la memoria implícita; y precisar las variables sociodemográficas y clínicas de estos pacientes.

Antes de iniciar el estudio y con la bibliografía disponible nos planteamos una serie de hipótesis previas: Las personas con más años de evolución del trastorno psicótico presentan mayor deterioro cognitivo y peor memoria explícita; la memoria implícita se mantiene estable independientemente de los años de evolución de la enfermedad; existe una correlación negativa entre el deterioro cognitivo y la memoria explícita; mientras que existe una correlación nula entre el deterioro cognitivo y la memoria implícita.

\section{METODOLOGÍA}

Para nuestro objetivo general, nuestra investigación consiste en un estudio de carácter descriptivo, observacional, transversal; cuya variable independiente (evolución del trastorno psicótico) presenta tres niveles (0-5 años, 10-15 años, 25-35 años) y cuyas variables dependientes son la memoria (implícita/explícita) y el 
deterioro cognitivo. Para nuestros objetivos específicos se utilizará un diseño de tipo correlacional (el cual se llevará a cabo una vez obtenidos los resultados preliminares y se haya aumentado la muestra del estudio). Para finalizar se han precisado las variables sociodemográficas de estos pacientes.

La población de estudio se extrajo de los dispositivos de salud mental que componen la Unidad de Gestión Clínica del Hospital Universitario Virgen del Rocío (Sevilla), concretamente en las Unidades de Salud Mental Comunitaria (USMC), Hospital de Día (HDSM) y Comunidad terapéutica Bermejales.

Como criterios de inclusión destacan: Diagnóstico trastorno psicótico según CIE-10; edad entre 18-65 años, cuyos años de evolución de la enfermedad se encuentren dentro de los tres grupos antes mencionados; aceptación voluntaria para tomar parte del estudio (consentimiento informado); pertenecer al área de salud del Hospital Virgen del Rocío (Sevilla).

Como criterios de exclusión destacan: Menores de edad; personas con Discapacidad intelectual moderada o grave evidenciada; no acepten participar en el estudio; conducta heteroagresiva; enfermedades cerebrovasculares, y enfermedades sistémicas y degenerativas como la demencia y el Parkinson.

El tamaño de la muestra constituye un estudio piloto con 20 sujetos repartidos entre los tres grupos (0-5 años; 10-15 años; 25-35 años). Una vez obtenidos y analizados los resultados preliminares se pretende aumentar la muestra realizando un cálculo exhaustivo para el tamaño de la misma. Todos los participantes dieron su consentimiento por escrito previo a su inclusión.

Las variables utilizadas son las siguientes: Evolución del Trastorno Psicótico con tres niveles (0-5 años; 10-15 años; 25-35 años), que constituye la variable independiente. Como variables dependientes disponemos de:

Memoria Implícita: Prueba de compleción de raíces de palabras (tarea de priming de raíces de palabras) $6,7,8,14$.

Memoria Explícita: Prueba de recuerdo señalado de raíces de palabras ${ }^{6,7,14}$.

Los estímulos y materiales necesarios para la prueba de memoria implícita y explícita son los siguientes: Se prepararán en hojas diferentes las cuatro listas de 20 palabras cada una. Se deben contrabalancear las listas en función de las cuatro condiciones: Tipo de tarea (explícita e implícita), tipo de estímulo (estudiado y no estudiado). De este modo todas las listas tendrán las mismas oportunidades de aparecer en las diferentes condiciones. Se asignarán un número a cada lista (1, 2, 3, y 4, por ejemplo); las palabras que se van a utilizar como estímulos se habrán aleatorizado. Las listas 1 y 2 se utilizarán para evaluar la memoria implícita mientras que las listas 3 y 4 se usarán para evaluar la memoria explícita. Cada participante realizará las tareas experimentales de manera individual. La tarea de memoria implícita siempre deberá ser la primera que deberán realizar todos los participantes para evitar la contaminación de la memoria explícita.

Cálculo de la puntuación: Para calcular la puntuación de cada participante en cada prueba de memoria deberá contar el número de aciertos obtenidos. En la prueba implícita, deberá contabilizar el número de raíces completadas con palabras que 
coincidan con las palabras de la lista estudiada, descontando el número de raíces completadas con palabras distintas a las presentadas en la fase de estudio. En la prueba explícita, deberá contar el número de raíces completadas con palabras de la lista estudiada descontando las raíces completadas con palabras distintas a las presentadas en la fase de estudio.

Para calcular la línea-base, calcule el número de raíces de palabras correspondientes a la lista no estudiada que el participante completó precisamente con las palabras contenidas en dicha lista sin haber sido presentadas en la fase de estudio. Haga lo mismo con la prueba explícita.

Una vez rellenados los resultados de cada participante en la prueba implícita en proporciones, la diferencia entre los valores de las proporciones (raíces completadas con palabras de la lista estudiada y las raíces completadas con palabras de la lista no estudiada) es el priming obtenido por cada participante; por consiguiente se obtendrá el valor medio obtenido por el grupo.

Para saber si en realidad existe memoria implícita deberá realizar la prueba estadística de contraste de medias (la t de Student). Si el resultado de la prueba estadística indica que la proporción media de raíces completadas con palabras estudiadas es significativamente superior a la proporción media de raíces completadas con palabras de la lista no estudiada y que las diferencias entre ambos valores no se debe al azar sino que son diferencias verdaderas con una probabilidad inferior al 0.05 ( $p<0.05)$ podrá concluir que existe priming. Esto es, los participantes en su estudio se han visto beneficiados por el hecho de haber estudiado en la fase de codificación una serie de estímulos, como es simplemente contar el número de letras que componen las palabras, produce facilitación o priming. En este caso, los participantes se habrán beneficiado con la presentación de las palabras en la primera fase del experimento.

Otra de las variables medidas hace referencia al deterioro cognitivo, para el que se utilizará el test de Pfeiffer (versión española) 10-11: Población diana: población general. Se trata de un cuestionario heteroadministrado que consta de 10 ítems. El punto de corte está en 3 o más errores, en el caso de personas que al menos sepan leer y escribir y de 4 o más para los que no. A partir de esa puntuación existe la sospecha de deterioro cognitivo.

También se medirá en consumo de tóxicos (historia clínica de consumo de tóxicos), junto a las variables sociodemográficas de los integrantes del estudio.

Consideraciones éticas: El estudio se ha desarrollado en todo momento siguiendo los principios éticos recogidos en la declaración de Helsinki, incluyendo la solicitud de Consentimiento informado a todos los pacientes que se incluirán en el mismo. Los pacientes fueron informados de la voluntariedad en la participación en el estudio y la correspondiente confidencialidad.

Los registros y el consentimiento informado de los pacientes están custodiados por el investigador principal; identificados por el número de caso que se le asigne, cumpliendo la legislación sobre protección de datos española (Ley orgánica 15/1999 de 13 de Diciembre). 


\section{RESULTADOS}

La media de edad del grupo de 0-5 años de evolución de la enfermedad fue de 23,44 años, para el de 10-15 años de evolución fue de 36.16 años y para el de 25-35 años de evolución de la enfermedad fue de 49.40 años. En el primer grupo hubo 5 hombres y 4 mujeres, en el segundo grupo 3 hombres y 3 mujeres, y en el tercer grupo 2 hombres y 3 mujeres. La distribución de las variables nivel de estudios (sin estudios, primarios, secundarios/FP, estudios universitarios), estado civil (soltero, casado, divorciado o viudo), situación laboral (empleado / desempleado / jubilado / incapacitado / estudiante) y consumo de tóxicos se describen a continuación:

1. Variables sociodemográficas: Nivel de estudios / Estado civil.

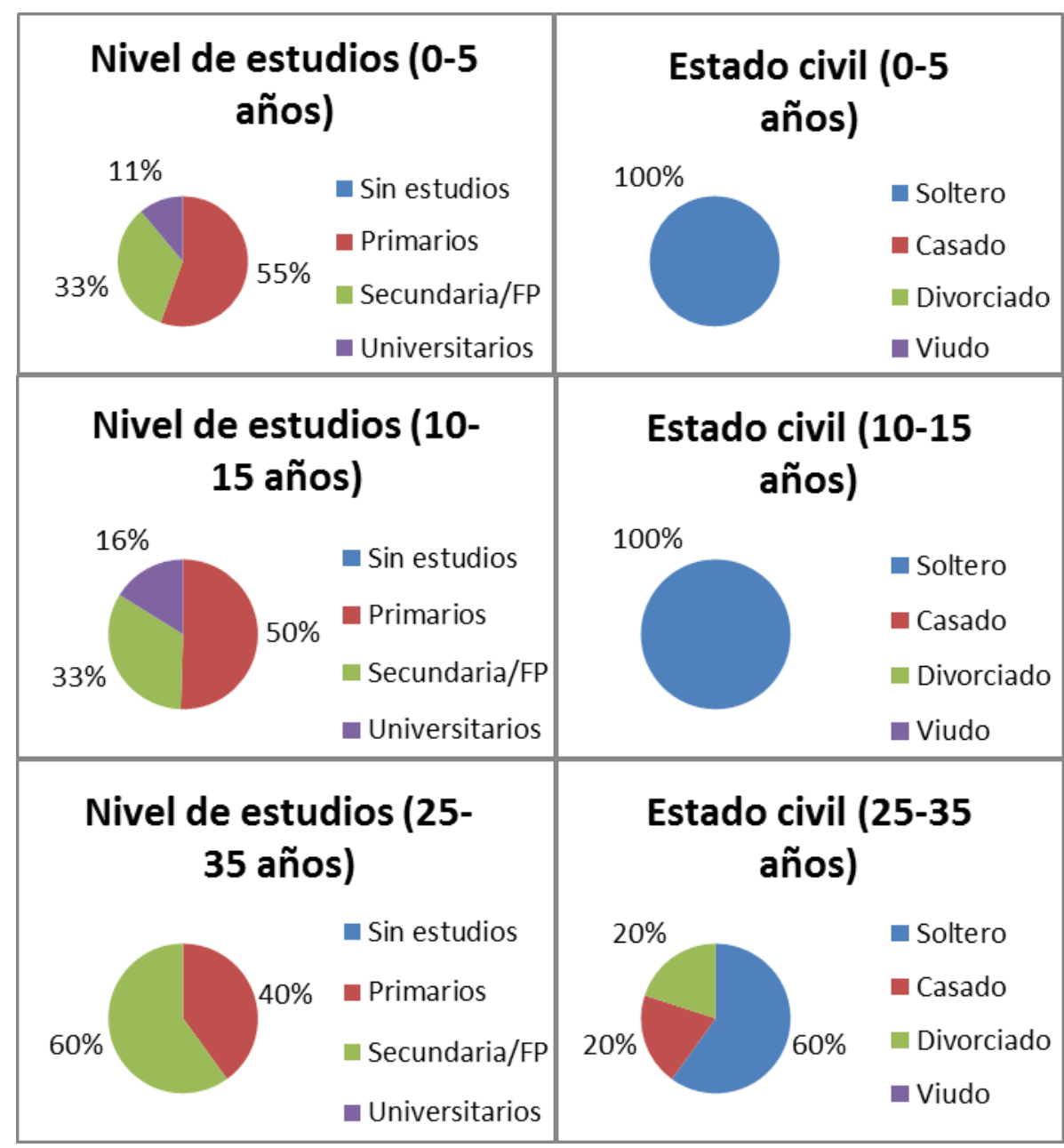

2. Variables sociodemográficas: Situación laboral / Consumo de tóxicos.

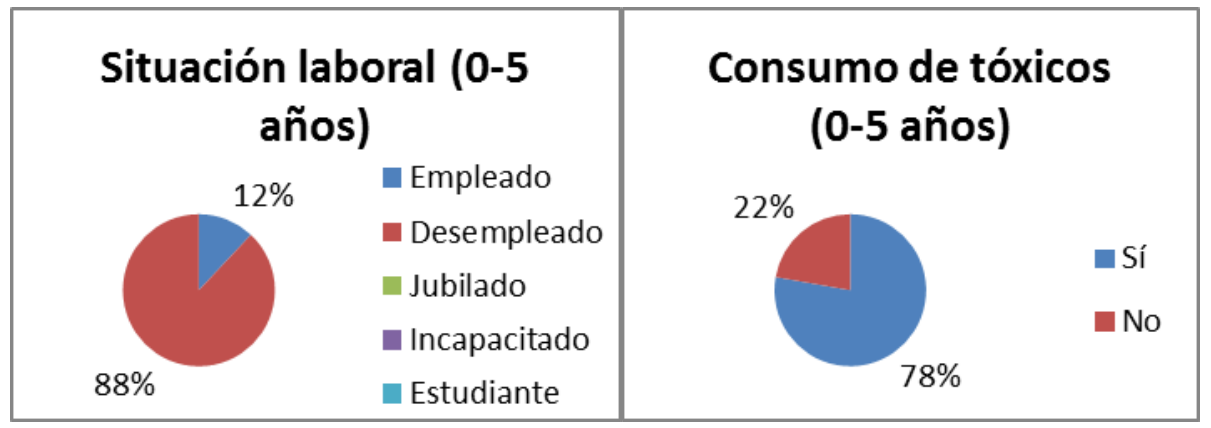




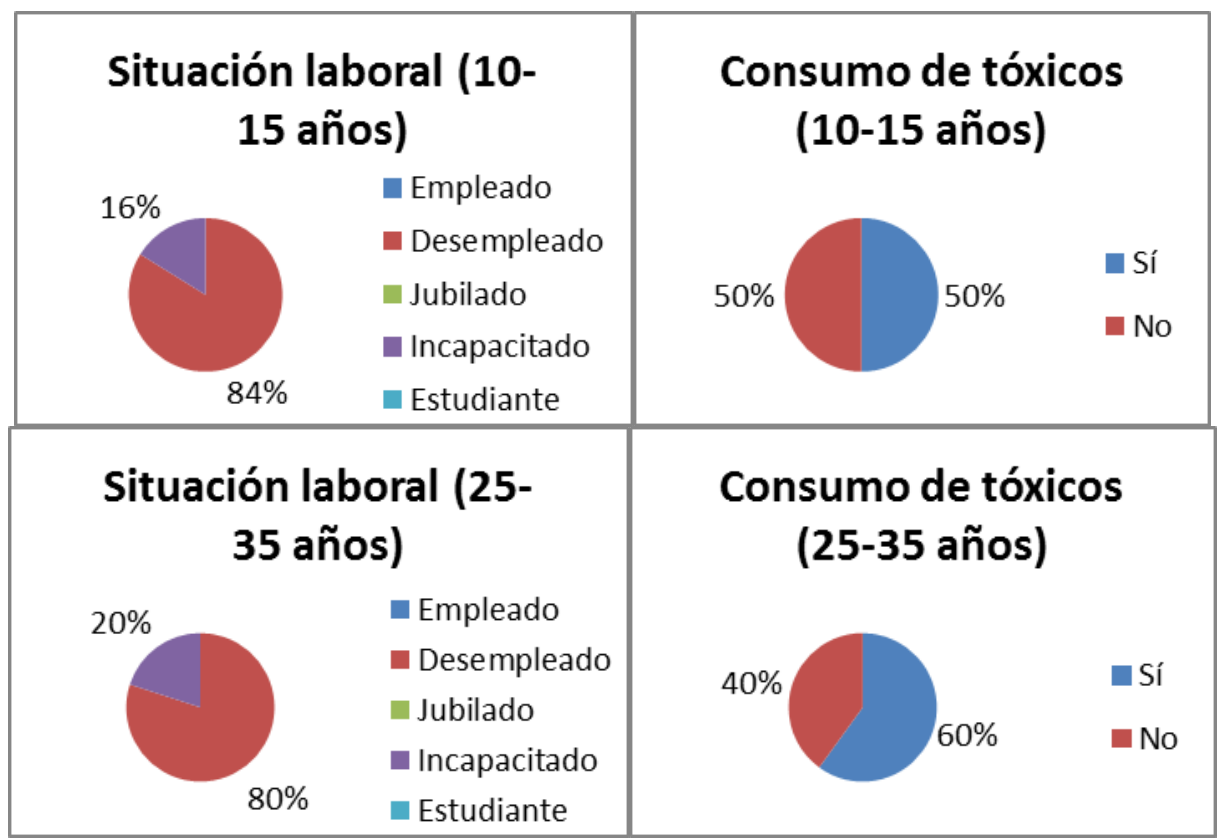

3. Resultados obtenidos respecto al cribado de deterioro cognitivo.

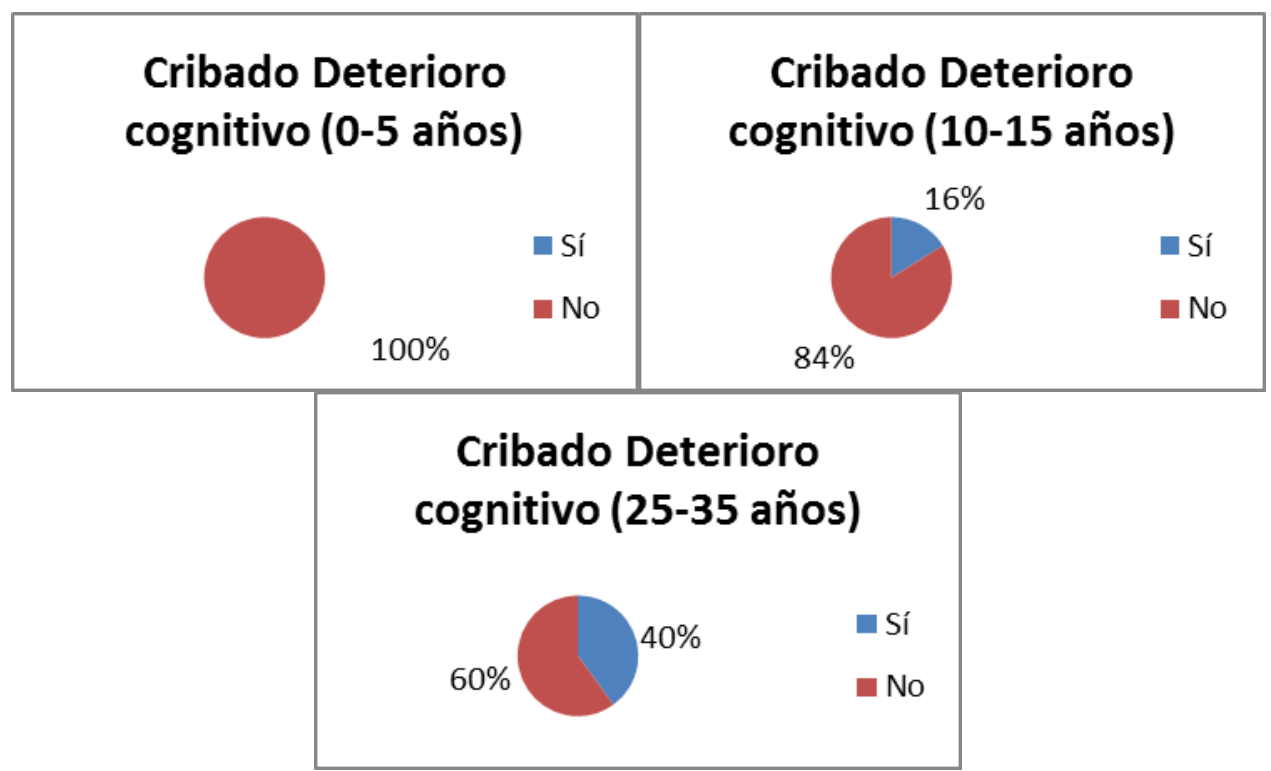

Los resultados obtenidos para la memoria implícita y memoria explícita para los tres grupos, se presentan en tabla I, II, III, IV, V y VI :(págs.14-19). 


\begin{tabular}{|c|c|c|c|}
\hline \multicolumn{4}{|c|}{$\begin{array}{c}\text { TABLA I *: PRIMER GRUPO (0-5 AÑOS DE EVOLUCIÓN DE LA ENFERMEDAD) } \\
\text { Prueba de priming de raíces de palabras (memoria implícita) }\end{array}$} \\
\hline Participante $n^{\circ}$ & \begin{tabular}{|c|} 
Proporción de raíces \\
completadas con palabras \\
estudiadas
\end{tabular} & $\begin{array}{l}\text { Proporción de raíces completadas con } \\
\text { palabras no estudiadas }\end{array}$ & Priming \\
\hline 1 & 0,35 & 0,15 & 0,2 \\
\hline 2 & 0,5 & 0,2 & 0,3 \\
\hline 3 & 0,4 & 0,1 & 0,2 \\
\hline 4 & 0,55 & 0,1 & 0,45 \\
\hline 5 & 0,3 & 0,2 & 0,1 \\
\hline 6 & 0,45 & 0,2 & 0,25 \\
\hline 7 & 0,65 & 0,15 & 0,5 \\
\hline 8 & 0,4 & 0,05 & 0,35 \\
\hline 9 & 0,45 & 0,15 & 0,3 \\
\hline $\begin{array}{l}\text { Proporción media } \\
\text { (desviación típica) }\end{array}$ & $0,45(0,10)$ & $0,14(0,05)$ & $0,29(0,12)$ \\
\hline $\begin{array}{l}1^{*} \text { : Tras la realizaciór } \\
\text { realizado la prueba e } \\
0,00000084965 \text {, para } \\
\text { Se concluye que exis }\end{array}$ & $\begin{array}{l}\text { los cálculos correspondie } \\
\text { ística de contraste de me } \\
\text { prueba con } 2 \text { colas de d } \\
\text { iming y por lo tanto mem }\end{array}$ & $\begin{array}{l}\text { ntes, y para saber si existe memoria imp } \\
\text { dias T de Student }(p<0,05) \text {, dando com } \\
\text { stribución y una varianza igual para las } \\
\text { oria implícita. }\end{array}$ & $\begin{array}{l}\text { lícita, se ha } \\
\text { o resultado } \\
2 \text { muestras. }\end{array}$ \\
\hline
\end{tabular}

\begin{tabular}{|c|c|c|c|}
\hline \multicolumn{4}{|c|}{$\begin{array}{c}\text { TABLA II: PRIMER GRUPO (0-5 AÑOS DE EVOLUCIÓN DE LA ENFERMEDAD) } \\
\text { Prueba de recuerdo señalado de raíces de palabras (memoria explícita) }\end{array}$} \\
\hline Participante no & Proporción de aciertos & $\begin{array}{c}\text { Proporción de falsas } \\
\text { alarmas }\end{array}$ & $\begin{array}{c}\text { Proporción de aciertos- } \\
\text { falsas alarmas }\end{array}$ \\
\hline 1 & 0,3 & 0,25 & 0,05 \\
\hline 2 & 0,5 & 0,1 & 0,4 \\
\hline 3 & 0,15 & 0,15 & 0 \\
\hline 4 & 0,2 & 0,1 & 0,1 \\
\hline 5 & 0,35 & 0 & 0,35 \\
\hline 6 & 0,4 & 0,1 & 0,3 \\
\hline 7 & 0,7 & 0,2 & 0,5 \\
\hline 8 & 0,45 & 0,15 & 0,3 \\
\hline 9 & 0,35 & 0,2 & 0,15 \\
\hline $\begin{array}{l}\text { Proporción media } \\
\text { (desviación típica) }\end{array}$ & $0,37(0,16)$ & $0,13(0,07)$ & $0,23(0,17)$ \\
\hline
\end{tabular}




\begin{tabular}{|c|c|c|c|}
\hline \multicolumn{4}{|c|}{ TABLA III *: SEGUNDO GRUPO (10-15 AÑOS DE EVOLUCIÓN DE LA ENFERMEDAD) } \\
Prueba de priming de raíces de palabras (memoria implícita)
\end{tabular}

\begin{tabular}{|c|c|c|c|}
\hline \multicolumn{4}{|c|}{$\begin{array}{l}\text { TABLA IV: SEGUNDO GRUPO (10-15 AÑOS DE EVOLUCIÓN DE LA ENFERMEDAD) } \\
\text { Prueba de recuerdo señalado de raíces de palabras (memoria explícita) }\end{array}$} \\
\hline Participante $\mathrm{n}^{0}$ & Proporción de aciertos & $\begin{array}{l}\text { Proporción de falsas } \\
\text { alarmas }\end{array}$ & $\begin{array}{l}\text { Proporción de aciertos- } \\
\text { falsas alarmas }\end{array}$ \\
\hline 1 & 0,4 & 0,15 & 0,25 \\
\hline 2 & 0,35 & 0,15 & 0,2 \\
\hline 3 & 0,4 & 0,2 & 0,2 \\
\hline 4 & 0,5 & 0,2 & 0,3 \\
\hline 5 & 0,35 & 0,1 & 0,25 \\
\hline 6 & 0,7 & 0,5 & 0,2 \\
\hline $\begin{array}{l}\text { Proporción media } \\
\text { (desviación típica) }\end{array}$ & $0,45(0,13)$ & $0,21(0,14)$ & $0,23(0,04)$ \\
\hline
\end{tabular}




\begin{tabular}{|c|c|c|c|}
\hline \multicolumn{4}{|c|}{$\begin{array}{c}\text { TABLA V *: TERCER GRUPO (25-35 AÑOS DE EVOLUCIÓN DE LA ENFERMEDAD) } \\
\text { Prueba de priming de raíces de palabras (memoria implícita) }\end{array}$} \\
\hline Participante $n^{0}$ & $\begin{array}{c}\text { Proporción de raíces } \\
\text { completadas con palabras } \\
\text { estudiadas }\end{array}$ & $\begin{array}{l}\text { Proporción de raíces completadas con } \\
\text { palabras no estudiadas }\end{array}$ & Priming \\
\hline 1 & 0,35 & 0,05 & 0,3 \\
\hline 2 & 0,3 & 0 & 0,3 \\
\hline 3 & 0,3 & 0,2 & 0,1 \\
\hline 4 & 0,35 & 0,05 & 0,3 \\
\hline 5 & 0,25 & 0,15 & 0,1 \\
\hline \begin{tabular}{|l|} 
Proporción media \\
(desviación típica)
\end{tabular} & $0,31(0,04)$ & $0,09(0,08)$ & $0,22(0,10)$ \\
\hline \multicolumn{4}{|c|}{$\begin{array}{l}5^{\star} \text { : Tras la realización de los cálculos correspondientes, y para saber si existe memoria implícita, se } \\
\text { ha realizado la prueba estadística de contraste de medias T de Student }(p<0,05) \text {, dando como } \\
\text { resultado } 0,000697661 \text {, para una prueba con } 2 \text { colas de distribución y una varianza igual para las } 2 \\
\text { muestras. } \\
\text { Se concluye que existe priming y por lo tanto memoria implícita. }\end{array}$} \\
\hline
\end{tabular}

\begin{tabular}{|c|c|c|c|}
\hline \multicolumn{4}{|c|}{$\begin{array}{l}\text { TABLA VI: TERCER GRUPO (25-35 AÑOS DE EVOLUCIÓN DE LA ENFERMEDAD) } \\
\text { Prueba de recuerdo señalado de raíces de palabras (memoria explícita) }\end{array}$} \\
\hline Participante $\mathrm{n}^{0}$ & Proporción de aciertos & $\begin{array}{l}\text { Proporción de falsas } \\
\text { alarmas }\end{array}$ & $\begin{array}{l}\text { Proporción de aciertos- } \\
\text { falsas alarmas }\end{array}$ \\
\hline 1 & 0,2 & 0,15 & 0,05 \\
\hline 2 & 0,3 & 0,25 & 0,05 \\
\hline 3 & 0,3 & 0,25 & 0,05 \\
\hline 4 & 0,2 & 0,2 & 0 \\
\hline 5 & 0,2 & 0,15 & 0,05 \\
\hline $\begin{array}{l}\text { Proporción media } \\
\text { (desviación típica) }\end{array}$ & $0,24(0,05)$ & $0,20(0,05)$ & $0,04(0,02)$ \\
\hline
\end{tabular}

\section{DISCUSIÓN}

Como puede observarse se obtienen proporciones medias mayores en los dos primeros grupos (0-5 años de evolución del Trastorno psicótico / 10-15 años de evolución del Trastorno psicótico) que en el tercer grupo (25-35 años de evolución del Trastorno psicótico) para los resultados de memoria explícita (proporción de aciertos/ proporción de falsas alarmas/ proporción de aciertos-falsas alarmas). Destacar que estos resultados se asocian con la bibliografía encontrada sobre la memoria explícita en esquizofrenia, destacando que el deterioro de la misma se encuentra asociado a la cronicidad de la enfermedad (13); es necesario mencionar que el déficit en la memoria verbal declarativa episódica parece ser el hallazgo más consistente ${ }^{(24)}$. 
Respecto a los resultados de la memoria implícita, tras la realización de los cálculos correspondientes (prueba T-Student), se observa la existencia de priming (memoria implícita) en los tres grupos de pacientes (se ha visto beneficiados por el hecho de haber estudiado en la fase de codificación estimular). También es necesario destacar cómo la memoria implícita se mantiene estable en los tres grupos a pesar de la presencia de deterioro cognitivo. La existencia de priming también se ve relacionada con los resultados en un estudio en el que se midió la memoria implícita en enfermos amnésicos, depresivos y esquizofrénicos que presentaron una memoria explícita muy deteriorada y una memoria implícita equivalente o casi equivalente a la de los individuos sanos ${ }^{(17)}$.

En relación a las puntuaciones en el deterioro cognitivo, se observa cómo la puntuación positiva en el cribado de deterioro cognitivo es mayor a medida que aumentan los años de evolución de la enfermedad; observándose cómo en la primera muestra hay ausencia de deterioro cognitivo, en la segunda muestra existe un $16 \%$ de deterioro cognitivo; mientras que en la tercera muestra tenemos un $40 \%$ de deterioro cognitivo, la cual coincide con las puntuaciones medias más bajas en la memoria explícita. Sin embargo, diversos estudios concluyen que al analizar el rendimiento cognitivo del grupo de pacientes esquizofrénicos según los años de evolución de la enfermedad pudieron observar que el deterioro cognitivo en la esquizofrenia tiende a permanecer estable ${ }^{\text {(27). }}$. Los principales déficits cognitivos en esta patología hacen referencia a las funciones ejecutivas, la memoria declarativa (memoria explícita), la memoria de trabajo y la atención sostenida ${ }^{(34)}$.

Estos resultados preliminares realizados hasta el momento con el procedimiento de compleción de raíces de palabras con instrucciones implícitas y explícitas tienen el fin de evaluar estas dos formas de recuperación de la información verbal en un grupo de adultos jóvenes; es una de las pruebas más utilizadas para evaluar el priming de repetición. Es necesario mencionar que también puede usarse esta misma tarea (modificando las instrucciones) para evaluar la memoria explícita como hicieron Osorio et al., en este caso la prueba se denomina recuerdo señalado de raíces de palabras. La utilización de la misma información estimular en la prueba implícita y en la explícita tiene la ventaja de que lo único que cambia son las instrucciones proporcionadas por el experimentador a la hora de evaluar la memoria ${ }^{(7,15)}$.

Los resultados de este estudio son aplicables y contribuyen a diversas mejoras en nuestra práctica clínica habitual; pues aunque no se evalúen intervenciones, al confirmarse nuestras hipótesis expuestas podrían sugerir la génesis de distintas intervenciones y programas para la rehabilitación de la memoria de los pacientes con Trastorno Psicótico con el fin de actuar directamente sobre algunas variables medidas como puede ser la memoria implícita, la cual, aunque los resultados hallados en anteriores estudios sugieren que la memoria basada en la recuperación implícita es similar a sujetos sanos, siendo necesario medir y reforzar los resultados anteriores; la memoria explícita, que se encuentra deteriorada en su conjunto (tanto memoria episódica como semántica), y el deterioro de las funciones cognitivas característico de la amplia gama de los trastornos psicóticos. Estas intervenciones también contribuirán a la modificación de las capacidades cerebrales, de manera que favorezca la rehabilitación psicosocial con el objetivo de recuperar el funcionamiento social de las personas con enfermedades neuroconductuales, de modo que mejorará la eficiencia en la gestión del sistema sanitario. Se han diseñado programas de rehabilitación cognitiva en psicosis, como el REHACOP ${ }^{(29)}$, además de realizarse trabajos en los que se estudia la aplicación y eficacia de la rehabilitación neurocognitiva en la esquizofrenia, mientras que 
existen otros estudios ${ }^{(30)}$ que demuestran un cambio en la activación cerebral asociado a la intervención cognitiva en esta patología.

Este estudio presenta varias limitaciones: En primer lugar, consiste en un estudio piloto con una muestra pequeña de sujetos $(n=20)$, lo que limita el interés general del estudio para poder extrapolar los resultados dentro de la población de pacientes con Trastorno psicótico de nuestro ámbito en general.

Además, el diseño transversal del estudio examina asociaciones pero no de relaciones causales, donde un cambio en un factor influye directamente en un cambio en otro, por ello no podremos asegurar que los resultados de deterioro cognitivo actuarán como factor predisponente al deterioro en la memoria implícita o en la explícita, ya que no se valora el deterioro cognitivo previo a la aparición del deterioro de la memoria. Por esta misma razón, tampoco podremos asegurar que sea consecuencia del déficit en la memoria. Además las dificultades que se pudieran encontrar en cuanto a los resultados de deterioro cognitivo y memoria pueden estar determinadas por otras variables de confusión, tales como, la comorbilidad de estos sujetos con otra patología mental e incluso orgánica, presentar C.I. Borderline, la presencia de consumo de tóxicos, u otra explicación alternativa. Cabe recordar que el propósito del estudio es básicamente describir los resultados de memoria y deterioro cognitivo en función de la evolución de la enfermedad (Trastorno psicótico).

Es necesario que se tenga en cuenta la presencia de un posible sesgo de selección, ya que el procedimiento de selección de los pacientes (ya sea por tiempo o recursos disponibles) será consecutivo, no dependerá del azar, aunque se asegurará que en la inclusión de los sujetos no influyan las preferencias de los investigadores, siguiéndose el orden de las citas establecidas previamente por el profesional ajeno al estudio

Por todo esto, destacar la necesidad de la Enfermería en el abordaje de los pacientes con Trastorno Psicótico, más específicamente y como nos atañe en nuestro estudio, en nuestro ámbito profesional y más en concreto dentro de la Salud Mental, enfatizando la importancia de trabajar desde nuestra amplia gama de intervenciones, no únicamente sobre la memoria (deterioro de la memoria 00131), sino sobre el conjunto de déficits cognitivos que impiden su integración en la sociedad y el desarrollo de su vida de una manera adaptativa.

\section{CONCLUSIONES.}

Teniendo en cuenta los resultados del estudio podemos extraer una serie de conclusiones a raíz de la revisión bibliográfica inicial y de las hipótesis planteadas:

-Las personas con más años de evolución del trastorno psicótico presentan mayor deterioro cognitivo y puntuaciones medias menores en memoria explícita.

-La memoria implícita se mantiene estable independientemente de los años de evolución de la enfermedad.

-Los grupos con mayor deterioro cognitivo presentan puntuaciones medias menores en memoria explícita.

-La presencia de memoria implícita permanece estable independientemente de la puntuación en deterioro cognitivo. 


\section{REFERENCIAS}

(1) Ballesteros Jiménez S. Psicología de la memoria: Estructuras, procesos, sistemas. 1a ed. Madrid: Uned-Univérsitas; 2012.

(2) Piedra García LA. Propuestas de la memoria en psicología: un estado de la cuestión y sus implicaciones en la enseñanza universitaria. Ciencias económicas. 2011; 29

(1):259-274.

(3) W. Papez J. Brain Mechanisms and Intelligence; A Quantitative Study of Injuries to the Brain by K. S. Lashley. The American Journal of Psychology. 1931; 43 (3):527-529.

(4) Morris RGM. Hebb DO. The organization of behavior, Wiley: New York;1949. Brain Research Bulletin. 1999; 50 (5-6):437.

(5) Carrillo-Mora P. Sistemas de memoria: reseña histórica, clasificación y conceptos actuales. Primera parte: Historia, taxonomía de la memoria, sistemas de memoria de largo plazo: la memoria semántica. Salud Mental. 2010; 33:85-93.

(6) Redondo MT, Reales JM, Ballesteros S. Memoria implícita y explícita en mayores no dementes con trastornos metabólicos producidos por la diabetes mellitus tipo 2 . Psicológica. 2010; 31:87-108.

(7) Ruíz-Vargas JM, Cuevas I. Priming perceptivo versus priming conceptual y efectos de los niveles de procesamiento sobre la memoria implícita. Psicothema. 1999; 11(4):853871.

(8) Ballesteros Soledad, Manuel Reales J, Manga D. Memoria implícita y memoria explícita intramodal e intermodal: influencia de las modalidades elegidas y del tipo de estímulos. Psicothema. 1999; 11(4):831-851.

(9) Pérez Martínez VT. El deterioro cognitivo: una mirada previsora. Rev Cubana Med Gen Integr. 2005; 21(1-2).

(10) Erkinjuntti T, Sulkava R, Wikstrom J, Autio L. Short Portable Mental Status Question naire as a screening test for dementia and delirium amog the elderly. J Am Geriatr Soc. 1987; 35(5): 412-6.

(11) Martinez de la Iglesia J, Duenas Herrero R, Onis Vilches MC, Aguado Taberne C, Albert Colomer C, Luque R. Adaptación y validación al castellano del cuestionario de Pfeiffer (SPMSQ) para detectar la existencia de deterioro cognitivo en personas mayores de 65 años. Med Clin (Barc). 2001; 117(4): 129-34.

(12) Packard PA, Rodríguez-Fornells A, Stein LM, Nicolás B, Fuentemilla L. Tracking explicit and implicit long-lasting traces of fearful memories in humans. Neurobiol Learn Mem. 2014 Sep;116:96-104.

(13) CIE-10 Clasificación estadística internacional de enfermedades y problemas relacionados con la salud. $10^{\underline{a}}$ ed. rev. Washington: Organización Mundial de la Salud: 2003.

(14) Nestor PG, Kubicki M, McCarley RW, et al. Episodic Memory and Neuroimaging of Hippocampus and Fornix in Chronic Schizophrenia. Psychiatry Research-Neuroimaging. 2007; 155(1): 21-28.

(15) Mayas Arellano J. Procesos atencionales y sistemas de memoria en el envejecimiento [tesis doctoral]. Madrid: Departamento de Psicología Básica II, Facultad de Psicología, Universidad Nacional de Educación a Distancia; 2008.

(16) Ruíz-Vargas JM. Psicología cognitiva de la memoria. La memoria como una realidad integrada en múltiples sistemas, procesos y niveles de análisis. Importancia de los estudios experimentales para un mejor conocimiento de la memoria humana desde la perspectiva de las ciencias de la cognición. Anthropos editorial. 2000; 189-190.

(17) Martinena Palacio P, Blas Navarro J, Medina Pradas C, Baños Yeste I, Sabanés A, Vicens Vilanova J et al. Esquizotipia y memoria verbal en población general adolescente.

Psicothema. 2006. 18(3):439-446. 
(18) Schwartz BL, Vaidya CJ, Shook D, Deutsch SI. Neural basis of implicit memory for socio-emotional information in schizophrenia. Psychiatry Res. 2013 Apr; 206(2-3):173-80. (19) Marques VR, Spataro P, Cestari V, Sciarretta A, lannarelli F, Rossi-Arnaud C. Is conceptual implicit memory impaired in schizophrenia? Evidence from lexical decision and category verification. Cogn Neuropsychiatry. 2014 Sep; 26:1-12.

(20) Neill E, Rossell SL. Comparing implicit and explicit semantic access of direct and indirect word pairs in schizophrenia to evaluate models of semantic memory. Psychiatry Res. 2013 Feb; 205(3):199-204.

(21): Miyoshi K, Minamoto T, Ashida H. Relationships between priming and subsequent recognition memory. Springerplus. Sept 2014; 3:546.

(22): Keane MM, Cruz ME, Verfaellie M. Attention and implicit memory: priming-induced benefits and costs have distinct attentional requirements. Mem Cognit. 2014 Sep.

(23) Rund BR, Borg NE. Cognitive deficits and cognitive training in schizophrenic patients: a review. Acta Psychiatr Scand.1999; 100:85-95.

(24) Flashman LA, Green MF. Review of cognition and brain structure in schizophrenia: profiles, longitudinal course and effects of treatment. Psychiatr Clin N Am. 2004; 27:1-18. (25) Czepielewski LS, Massuda R, Goi P, Sulzbach-Vianna M, Reckziegel R, Costanzi M, Kapczinski F, Rosa AR, Gama CS. Verbal episodic memory along the course of schizophrenia and bipolar disorder: A new perspective. Eur Neuropsychopharmacol. 2014 Sept; 16.

(26) S. Eichemail T, Evan Nee D, Insel C, Malapani C, E. Smith E. Neural Correlates of Impaired Cognitive Control over Working Memory in Schizophrenia. Biological Psychiatry. 2014 Jul; 76 (2): 146-153.

(27) Ginarte Arias Y, Rivero Fernández T, Aguilera Reyes ML. Evaluación neuropsicológica en pacientes esquizofrénicos. Rev. Hosp. Psiquiátrico de la Habana. $2005 ; 2(3)$.

(28) Abadias ME, Chesa D, Izquierdo E, Fernández E, Sitjas M. Eficacia de la rehabilitación cognitiva en la esquizofrenia: una revisión. Rev. Asoc. Esp. Neuropsiq. 2003; 86:91-103.

(29) Ojeda N, Peña J, Bengoetxea E, García A, Sánchez P, Elizagárate E, et al. Evidencias de eficacia de la rehabilitación cognitiva en psicosis y esquizofrenia con el programa REHACOP. Rev Neurol. 2012; 54:577-86.

(30) Wykes T, Brammer M, Mellers J, Bray P, Reeder C, Williams C, et al. Effects on the brain of a psychological treatment: cognitive remediation therapy: functional magnetic resonance imaging in schizophrenia. Br J Psychiatry. 2002; 181: 144-52.

(31) M. Barch D, M. Sheffield J. Cognitive impairments in psychotic disorders: common mechanisms and measurement. World Psychiatry. Oct 2014; 13 (3):224-232.

(32) C. Gur R, E. Gur R. Memory in health and in schizophrenia. Dialogues Clin Neurosci. 2013 Dec; 15(4): 399-410.

(33) Harvey PD, Sharma T. Understanding and Treating Cognition in Schizophrenia. A Clinician's Handbook. London: Martin Dunitz; 2002.

(34) Harvey PD, Howanitz E, Parrella M, White L, Davidson M, Mohs RC, Hoblyn J, Davis KL. Symptoms, cognitive functioning, and adaptative skills in geriatric patients with lifelong schizophrenia: a comparison across treatment sites. Am J Psychiatry. 1998; 155:6-1080.

Recibido: 25 de septiembre 2014; Aceptado: 14 de noviembre 2014 
ISSN 1695-6141

๑ COPYRIGHT Servicio de Publicaciones - Universidad de Murcia 